\title{
Drive for Muscularity: Um Estudo Exploratório no Exército Brasileiro
}

\author{
Angela Nogueira Neves Betanho Campana ${ }^{1}$ \\ Universidade Sagrado Coração \\ Jairo José Monteiro Morgado \\ Instituto de Pesquisa da Capacitação Física do Exército \\ Fabiane Frota da Rocha Morgado \\ Universidade Estadual de Campinas \\ Mateus Betanho Campana \\ Hospital Infantil de Investigações Hematológicas Dr. Domingos A. Boldrini \\ Lucilene Ferreira \\ Universidade Sagrado Coração \\ Maria da Consolação Gomes Cunha Fernandes Tavares \\ Universidade Estadual de Campinas
}

\begin{abstract}
RESUMO - Drive for muscularity refere-se ao desejo de alcançar o corpo muscular ideal e ao grau de preocupação a respeito da musculatura. O objetivo do presente estudo foi investigar os traços de drive for muscularity em homens militares brasileiros. Dados de uma amostra não-probabilística de 652 homens foram analisados. Os resultados mostraram associações entre as variáveis de drive for muscularity e os hábitos de prática de atividade física, satisfação com a vida e com o corpo e ansiedade físico-social. Diferenças nos traços de drive for muscularity foram achados em relação ao status de relacionamento amoroso, segurança financeira e tempo de carreira no exército. Concluiu-se que o acompanhamento de drive for muscularity na formação do militar deveria fazer parte das rotinas de cuidados psicossociais.
\end{abstract}

Palavras-chave: imagem corporal, saúde do homem, militares

\section{Drive for Muscularity: An Exploratory Study in the Brazilian Army}

\begin{abstract}
Drive for muscularity refers to the desire to achieve an ideal muscular body, as well the degree of concern about increasing muscle mass. The aim of this research was to investigate drive for muscularity traits in men from the Brazilian army. Data from a non-probabilistic sample of 652 men were analyzed. Results showed associations among drive for muscularity variables and habits of physical exercise practice, life and body satisfaction, and social physique anxiety. Differences in drive for muscularity traits were also found accordingly with the romantic status, financial security and time career in the army. It was concluded that monitoring the drive for muscularity during military education should be part of the psychosocial care routines.
\end{abstract}

Keywords: body image, men's health, military

Drive for muscularity foi um construto estruturado no contexto da ampliação da pesquisa em imagem corporal do homem, quando a musculatura - seja sua definição, volume ou aparência - passou a ser considerada um aspecto central da imagem corporal do homem. Refere-se ao desejo de alcançar um corpo muscular idealizado, e ainda, ao grau de preocupação que o sujeito pode ter a respeito do aumento da sua musculatura. Refere-se também à necessidade de atender ao padrão de atratividade física masculina, no qual o sujeito mesomórfico, mais forte, mais musculoso e com menor percentual de gordura, é tido como atraente (McCreary \& Sasse, 2000; McCreary, Sasse, Saucier, \& Dorsch, 2004; Morrison, Morrison, \& McCann, 2006).

Nem sempre os comportamentos adotados para a aquisição de um corpo forte são saudáveis (Bahrke, 2007). Entre os comportamentos negativos associados ao drive for muscularity destacam-se o uso de esteróides anabolizantes, prática excessiva de exercícios resistidos, uso de diuréticos, checagem constante do volume muscular e evitação do corpo

1 Endereço para correspondência: Setor de Pós-Graduação - Fisioterapia. Rua Irmã Arminda, 10-50. Jardim Brasil, Bauru - SP. CEP 17011-160. E-mail: angelanneves@yahoo.com.br
- que por sua vez, reflete-se na esquiva de mostrar o corpo, na resistência em manter contato íntimo e por meio do uso de roupas largas (Pope et al., 2005; Pope, Phillips, \& Olivardia, 2000; Olivardia, 2007). Na dismorfia muscular, que é um quadro clínico de extrema insatisfação com a musculatura, com comprometimento nos aspectos sociais, emocionais e econômicos do sujeito, altos níveis de drive for muscularity são sistematicamente encontrados (Pope, Gruber, Choi, Olivardia, \& Phillips, 1997). Considerando a importância que a musculatura tem na avaliação geral de satisfação com o corpo (McCreary, 2011), os profissionais que trabalham diretamente com o exercício físico ou treinamento, seja no âmbito do lazer ou em atividades profissionais, devem ter conhecimento suficiente para oferecer ao seu aluno/ cliente um treinamento adequado quanto aos parâmetros do treinamento esportivo. Devem também ter uma atitude que valorize as potencialidades e que possibilite a vivência dos limites corporais, minimizando comparações com modelos midiáticos e evitando a supervalorização da aparência. Dessa forma, esses profissionais colaborarão para a manutenção de níveis mais baixos de drive for muscularity e, possivelmente, de menor incidência de quadros de dismorfia muscular. 


\section{Método}

No Brasil, a compreensão dos diferentes aspectos de drive for muscularity na população é relevante, haja vista a importância que a aparência do corpo musculoso tem entre os homens brasileiros (Goldenberg, 2002; Sabino, 2000). Os homens que servem ao Exército Brasileiro estão em um cenário particular. Reconhece-se a importância da aparência física na atratividade, que, como já dissemos, é um elemento central para o conceito de corpo do homem brasileiro. Todavia, não se pode ignorar que urge alcançar uma compreensão mais profunda do valor da musculatura nesse público específico, pois o padrão de força muscular e um porte físico mais proeminente podem ser considerados um dos pré-requisitos para o sucesso na carreira profissional.

Os resultados dos Testes de Aptidão Física anuais, em conjunto com os resultados de outras avaliações, são parâmetros considerados na classificação dos militares nas Escolas de Formação. Influenciam diretamente a carreira do militar, em atividades como a escolha das Armas e escolha da Unidade que o indivíduo irá servir após o curso, e farão parte da quantificação do mérito do militar (Morgado, 2012). Assim, para esses profissionais, a muscularidade vai além das questões relativas à aparência e à atratividade física. Versa sobre a virilidade, o valor como trabalhador, a masculinidade, as chances de êxito em combate e a capacidade de manter em segurança a própria vida, capacidades e qualidades estas que são também avaliadas em cada resultado de teste de desempenho físico.

Um corpo físico que remete a um homem viril e potente, capaz de defender a pátria, tem sua busca incentivada, como pode-se perceber da leitura do Manual de treinamento Militar do Estado Maior do Exército (EME, 2002), que determina padrões mínimos e avançados de aptidão física a serem alcançados, com a explicitação da importância de cada um deles na força.

Quando o militar não alcança um padrão aceitável em sua avaliação física periódica, é encaminhado para um especialista que realizará um treinamento específico, em complementação ao treinamento físico militar padrão, visando obter a suficiência no padrão exigido em sua função (EME, 2008).

Se, por um lado, essa vigília sobre o corpo e a forma física garantem que os militares tenham condições físicas para atuar nos segmentos que são designados, por outro lado, está associada a transtornos alimentares, ansiedade físico-social, insatisfação com o corpo, baixa autoestima (Carlton, Manos, \& Van Slyke, 2005; Gunderson, 1965; McNulty, 1997).

O sofrimento psíquico que esses profissionais podem experimentar em relação ao seu corpo, aliado à percepção dos riscos inerentes à sua própria profissão, pode desestabilizar emocionalmente o sujeito, impedindo-o de ter a melhor resposta em suas missões e, de uma forma mais ampla, ter uma qualidade de vida aceitável (Neves \& Mello, 2009).

Considerando a argumentação anterior, o objetivo deste trabalho foi colaborar para a melhor compreensão dos traços de drive for muscularity no Brasil, particulamente no Exército Brasileiro, uma população em que o exercício físico, o corpo musculoso e o desempenho físico são comumente associados à qualidade do indivíduo enquanto soldado.
Trata-se de um estudo transversal exploratório. Obedecendo à legislação de ética em pesquisa 196/96, o estudo iniciou-se apenas após recebimento do parecer favorável do Comité de Ética da Unicamp, registrado sob Protocolo $n^{\circ}$ 0828.0.146.000-08.

\section{Participantes}

O cálculo amostral foi feito considerando um nível de confiança de $95 \%$ e um intervalo de confiança de $4 \%$, através da fórmula

Número Amostral $=\left(Z^{2} * \mathrm{p} *(1-\mathrm{p})\right) / \mathrm{m}^{2}$

em que $Z=$ nível de confiança (no caso, 1,96 para 95\% do nível de confiança), $\mathrm{p}=$ valor padrão $0,5, \mathrm{~m}=$ intervalo de confiança ou margem de erro, expresso em decimais (no caso 0,04) (Magnani, 1999). Foi determinado número amostral mínimo de 600 participantes.

Uma amostra não-probabilística, de 654 homens militares foi recrutada no Curso de Formação de Sargentos do Exército Brasileiro de um quartel situado numa cidade do estado de Minas Gerais, dos quais 50\% eram ingressantes. A média de idade foi 19,57 anos $(\mathrm{DP}=3,01 ; \min =18, \max =39$ anos) e o índice de massa corporal (IMC) médio foi $23,06 \mathrm{~kg}$ / $\mathrm{m} 2(\mathrm{DP}=2,74 ; \min =15,74, \max =34,6)$. A grande maioria era fisicamente ativa $(79,8 \%)$ e praticava atividade física em intensidade pesada $(58,1 \%)$, com duração acima de 30 minutos por sessão $(64,2 \%)$, de três a cinco vezes por semana $(48,6 \%)$.

\section{Instrumentos}

Foram utilizados os seguintes instrumentos:

Versão Brasileira da Drive for Muscularity Scale - DMS (McCreary \& Sasse, 2000; versão para o Brasil: Campana, Tavares, Swami, \& Silva, 2013). A DMS foi elaborada para avaliar o desejo de ficar mais forte. As respostas estão dispostas numa Escala Likert de seis pontos $(1=$ sempre a $6=$ nunca) e todos os itens devem ter escore revertido para análise. O escore final é dado pela soma dos itens dos respectivos fatores e maiores escores indicam maior frequência de comportamentos voltados para obtenção de um corpo forte e maior direcionamento ao corpo musculoso. O modelo original, com dois fatores, chamados Orientação à Musculatura (DMS-OM) e Comportamentos relacionados ao Desenvolvimento da Musculatura (DMS-CM), obteve melhor ajuste na versão brasileira da DMS (RMSEA $=0,067$, $\mathrm{GFI}=0,99, \mathrm{AGFI}=0,99, \mathrm{NFI}=0,99, \mathrm{CFI}=0,99, \mathrm{NNFI}=0,99)$ com valores de confiabilidade adequados (Campana et al., 2013). Nesta amostra, o valor do alpha de Cronbach para o fator 1 , DMS-OM, foi $\alpha=0,87$ e para o fator 2 , DMS-CM, foi $\alpha=0,86$.

Versão Brasileira da Swansea Muscularity Attitudes Questionnaire - SMAQ (Edwards \& Launder, 2000; versão para o Brasil: Campana et al., 2013). A SMAQ foi criada para avaliar as preocupações e atitudes relacionadas à musculatura. A versão brasileira, diferente da original, tem uma estrutura fatorial de três fatores, com bons índices de 
ajuste $(\mathrm{RMSEA}=0,050 ; \mathrm{GFI}=0,99 ; \mathrm{AGFI}=0,99 ; \mathrm{NFI}=0,99$; $\mathrm{CFI}=0,99$; NNFI $=0,99)$. Os valores de confiabilidade composta (CC) para os fatores Investimento na Musculatura (SMAQ-I; $\mathrm{CC}=0,90)$, Valor Atribuído à Musculatura (SMAQ-VM; $\mathrm{CC}=0,86$ ) e Masculinidade e Muscularidade (SMAQ-MM; $C C=0,74$ ) foram adequados. As respostas estão dispostas numa Escala Likert de sete pontos ( $1=$ concordo totalmente a $7=$ discordo totalmente) e o escore final á dado pela soma dos escores do itens nos seus respectivos fatores. Maiores escores indicam maior investimento, valor e associação da musculatura com masculinidade. Nessa amostra, os valores de alpha de cronbach para os fatores SMAQ-I, SMAQ-VM e SMAQ-MM foram $\alpha=0,89,0,83 \mathrm{e}$ 0,75 , respectivamente.

Versão Brasileira da Masculine Body Ideal Distress Scale - MBIDS (Kimmel \& Mahalick, 2004; versão para o Brasil: Campana et. al., 2013). A MBIDS foi desenvolvida para avaliar o quanto um homem se angustia por não ter um corpo que responda aos padrões de beleza. A versão brasileira da MBIDS é composta por seis itens, organizados em um único fator (RMSEA $=0,053$, GFI $=1, \mathrm{AGFI}=0,99$, $\mathrm{NFI}=1, \mathrm{CFI}=1, \mathrm{NNFI}=1$ ), e apresenta boas evidências de confiabilidade, sendo $\alpha=0,84$ e $C C=0,82$. As respostas estão dispostas numa Escala Likert de quatro pontos, que variam de 1=nada desconfortável a 4=muito desconfortável. O resultado da escala é dado pela soma de todos os itens. Altos escores indicam alto desconforto por estar longe do corpo ideal/ modelo de beleza. Nesta amostra, o valor de alpha de Cronbach para o escore da MBIDS foi $\alpha=0,82$.

Versão Brasileira da Social Physique Anxiety Scale SPAS (Hart, Leary, \& Rejesky, 1989; versão para o Brasil: Campana, Tavares, \& Silva, 2011). A SPAS foi criada para avaliar o grau de ansiedade que uma pessoa experimenta quando outros estão observando ou avaliando o seu corpo. No Brasil, a estrutura fatorial com melhor ajuste foi a unidimensional, com 10 itens (RMSEA $=0,045$, GFI $=0,99$, $\mathrm{AGFI}=0,99, \mathrm{NFI}=1, \mathrm{CFI}=1, \mathrm{NNFI}=0,99)$. Quanto à confiabilidade, o teste de alpha de Cronbach foi $\alpha=0,82$ e a confiabilidade composta $\mathrm{CC}=0,81$ (Campana et al., 2011). As opções de respostas estão dispostas numa Escala Likert de cinco pontos $(1=$ nada parecido comigo a $5=$ tudo a ver comigo). Os itens 1 e 5 são reversos. O escore final da escala é dado pela soma de todos os itens. Quanto maior o escore, maior é o grau de ansiedade frente ao olhar do outro. O valor de alpha de Cronbach para o escore final da SPAS nesta amostra foi $\alpha=0,83$.

Questionário demográfico. Nesse questionário, os participantes autorreportaram informações demográficas e antropométricas, como por exemplo, idade, peso, altura. Responderam também a três perguntas diretas: Numa escala de um a 10 (sendo 1 "nem um pouco satisfeito" e 10 "bastante satisfeito"), o quão satisfeito você está com a sua vida?"; "Numa escala de um a dez (sendo 1 "nem um pouco satisfeito" e 10 "bastante satisfeito"), o quão satisfeito você está com o seu corpo?; O quão seguro financeiramente você se sente (sendo $1=$ inseguro, $2=$ um pouco inseguro, $3=$ um pouco seguro e $4=$ seguro)?

\section{Procedimento}

Os pesquisadores visitaram cada grupamento do quartel, explicando oralmente os objetivos da pesquisa. Em seguida, colocaram-se à espera dos participantes voluntários em uma sala previamente reservada, na qual foram organizadas mesas individuais para o preenchimento do pacote de questionários, todos autopreenchíveis. A sala tinha capacidade para receber até 20 participantes de forma concomitante. Após a leitura do Termo de Consentimento Livre e Esclarecido, aqueles que concordaram e o assinaram, receberam o pacote com os questionários, que foram preenchidos de forma anônima. Todos os questionários foram preenchidos na presença dos pesquisadores, garantindo a independência das respostas. $\mathrm{O}$ tempo de preenchimento foi de aproximadamente 20 minutos.

Nenhum dos indivíduos convidados recusou participar da pesquisa. A participação foi totalmente voluntária e os participantes não receberam nenhuma forma de remuneração.

\section{Análise de dados}

Inicialmente, o teste de normalidade de KolmogorovSmirnov foi usado para verificar a aderência dos dados à distribuição normal. Como constatou-se que os dados eram não paramétricos, o teste de correlação de Spearman foi utilizado para investigação das associações entre as variáveis e o teste de Mann-Whitney para investigar diferenças entre grupos, formados de acordo com as variáveis categóricas do estudo. Face à natureza não paramétrica da distribuição dos dados, o que limita a realização de uma análise de regressão, optou-se por uma abordagem multivariada descritiva e não inferencial, exploratória em essência, a análise de cluster. Esta é uma técnica estatística multivariada que classifica objetos (respondentes, variáveis), separando grupos com homogeneidade interna e, ao mesmo tempo, dissemelhantes entre si (Hair, Anderson, Tatham, \& Black, 2009). Adotou-se a seleção listwise, para evitar viés nos resultados, e nível de significância de $95 \%$, sendo que as correlações significantes a 99\% também foram consideradas. O software SPSS, versão 15 , foi usado em todas as análises.

\section{Resultados}

\section{Associações entre as variáveis}

Inicialmente, foi realizada a análise de correlação bivarida entre os escores fatores da DMS e da SMAQ, do escore da MBIDS, da SPAS e variáveis demográficas contínuas, como idade, intensidade, duração e frequência da atividade física e nível de satisfação com a vida e com o corpo, para o grupo de alunos ingressantes e veteranos separadamente. As caracteristicas descritivas (mediana e amplitude) de todos os participantes da amostra estão na Tabela 1, assim como as correlações encontradas para os alunos ingressantes e os veteranos.

Para os alunos ingressantes no Curso de Formação de Sargentos, não houve correlações significantes entre a idade 


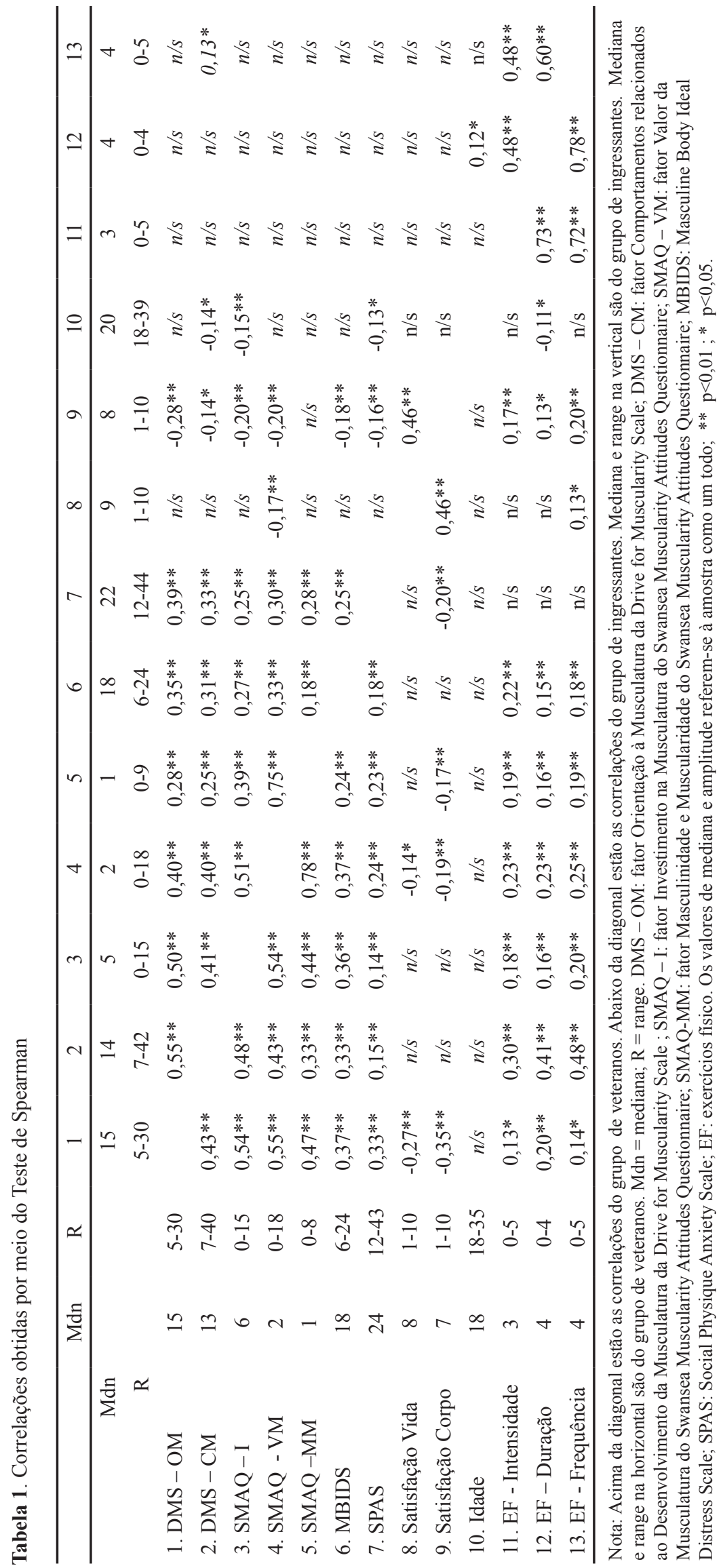


e os escores dos fatores e das escalas em análise. Já para os veteranos, a idade apresentou correlações muito fracas com o escore do fator DMS-CM, com o fator SMAQ-I e com o escore da SPAS. Padrão similar ocorreu com a variável satisfação com a vida, em que apenas uma associação muito fraca com o fator SMAQ-VM foi verificada.

Para os alunos ingressantes, o escore da medida direta de satisfação com a vida correlacionou-se com o fator DMS-OM e com o fator SMAQ-VM. Tanto para os ingressantes quanto para os veteranos, houve uma correlação moderada da medida direta de satisfação com a vida com a de satisfação com o corpo. Correlações moderadas a fracas foram encontradas entre a medida direta de satisfação com o corpo e todos os fatores da DMS, com os fatores SMAQ-I e SMAQ-VM, com o escore da SPAS e da MBIDS e com o fator OM-DSM, para os alunos veteranos. Para os alunos ingressantes, correlações mais fortes foram encontradas entre a medida direta de satisfação com o corpo e os fatores DMS-OM, SMAQ-VM, SMAQ -MM e SPAS.

Em relação ao exercício físico, para os alunos ingressantes, a intensidade, frequência e duração dos exercícios correlacionaram-se com todos os escores dos fatores da DMS, da SMAQ e com os escores da MBIDS. Já para os alunos veteranos, não houve correlação significante entre as variáveis caracterizadoras do exercício físico e os escores dos fatores e das escalas em estudo.

Os fatores das escalas DM e SMAQ e os escores da MBIDS e SPAS, por sua vez, associaram-se todos positivamente entre si em ambos os grupos, em correlações que variaram de mais fracas a fortes.

\section{Interdependência entre as variáveis}

Determinou-se que a medida de distância euclidiana seria usada para inferir as (dis)semelhanças entre as variáveis, por ser uma das mais usadas na análise de cluster (Malhotra,
1999). Por causa do caráter exploratório deste estudo e por não haver, a priori, o estabelecimento de relações causais em potencial e, por conseguinte, do número de clusters, os sete métodos de aglomeração hierárquicos foram testados para verificar qual separaria melhor as variáveis. O método hierárquico de Ward foi o que mostrou melhor separação das variáveis e os resultados aqui apresentados dizem respeito àqueles gerados pela análise com esse método hierárquico de aglomeração. O dendograma da solução da análise do cluster indica a existência de três clusters distintos (ver Figura 1).

O primeiro grupo foi formado pelas variáveis caracterizadoras da atividade física (intensidade, duração e frequência) praticada pelos participantes, pelo fator SMAQVM e pelo fator SMAQ-MM. O segundo grupo foi formado pelas medidas diretas de satisfação com a vida, satisfação com o corpo e pelo fator SMAQ-I. O terceiro grupo foi formado pelas variáveis MBIDS, SPAS, e pelos fatores DMS-OM e DMS-CM.

\section{Diferenças entre grupos: sem relacionamento afetivo vs. comprometidos}

Foram consideradas pessoas "sem relacionamento afetivo" aqueles participantes que se declaram solteiros sem namorada(o), viúvos e divorciados/separados. Já o grupo de pessoas "comprometidas" foi formado por participantes solteiros, mas que estavam namorando, e aqueles casados.

Os resultados sugerem uma diferença significante no fator SMAQ-I $(\mathrm{U}=46637 ; \mathrm{p}=0,03 ; \mathrm{r}=0,08)$, no fator SMAQVM (U=46183,5; $\mathrm{p}=0,02 ; \mathrm{r}=0,09)$ e no fator SMAQ-MM $(\mathrm{U}=46462,5 ; \mathrm{p}=0,02 ; \mathrm{r}=0,09)$, sendo que em todos esses casos, os voluntários que não estavam namorando, casados ou noivos tinham maiores valores referentes a essas atitudes. Quanto ao escore da SPAS, da MBIDS e dos fatores DMSCM e DMS-OM não houve diferença estatística entre os grupos (ver Tabela 2).

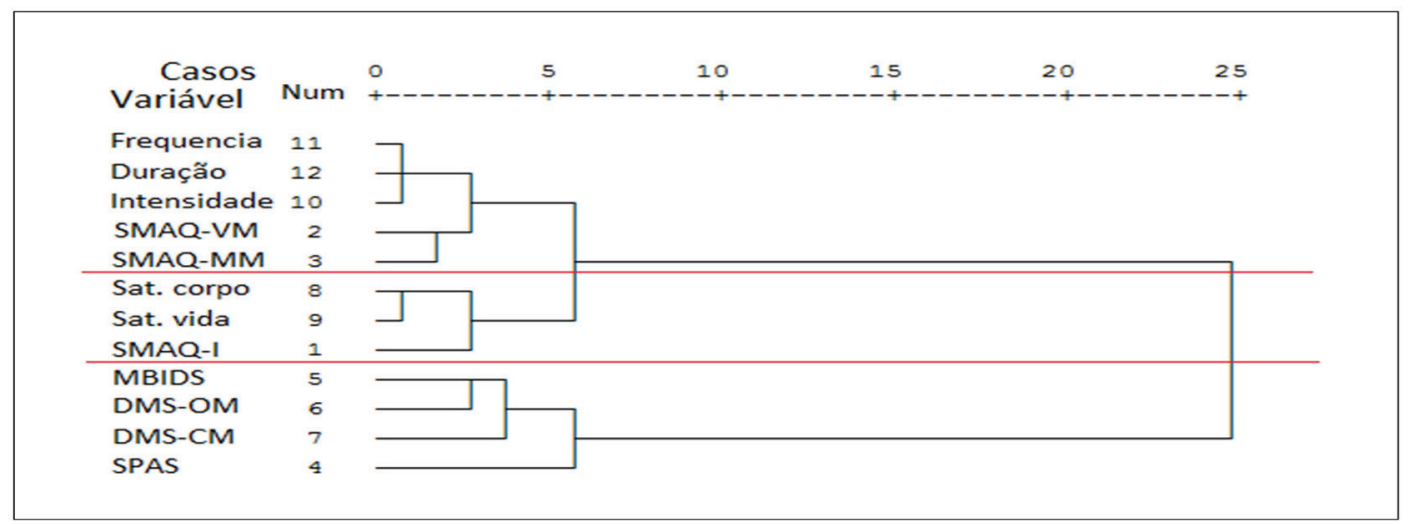

Figura 1. Dendograma da análise de cluster (Linhas em vermelho indicam separação dos clusters. DMS - OM: fator Orientação à Musculatura da Drive for Muscularity Scale; DMS - CM: fator Comportamentos relacionados ao Desenvolvimento da Musculatura da Drive for Muscularity Scale; SMAQ - I: fator Investimento na Musculatura do Swansea Muscularity Attitudes Questionnaire; SMAQ - VM: fator Valor da Musculatura do Swansea Muscularity Attitudes Questionnaire; SMAQ-MM: fator Masculinidade e Muscularidade do Swansea Muscularity Attitudes Questionnaire; MBIDS: Masculine Body Ideal Distress Scale; SPAS: Social Physique Anxiety Scale; Sat.corpo: satisfação com o corpo; Sat. vida = satisfação com a vida). 
Diferenças entre grupos: inseguros vs. seguros financeiramente

As informações a respeito da segurança financeira foram geradas por meio da pergunta direta "O quão seguro financeiramente vocês se sente (sendo 1=inseguro e 4=seguro)?". Os resultados deste estudo apontam que a segurança financeira pode ser um fator de importância também para drive for muscularity, apontando diferenças significantes em quatro das sete variáveis investigadas, a saber: SMAQ-VM (U=41811; $\mathrm{p}=0,002 ; \mathrm{r}=0,12)$, SMAQMM (U=44273; $\mathrm{p}=0,03 ; \mathrm{r}=0,08), \mathrm{DMS}-\mathrm{OM}(\mathrm{U}=40902,5$; $\mathrm{p}=0,001 ; \mathrm{r}=0,18)$ e SPAS $(\mathrm{U}=38131,5 ; \mathrm{p}<0.001 ; \mathrm{r}=0,13)$ (ver Tabela 3).

Tabela 2. Diferença entre grupos: sem relacionamento afetivos vs. comprometidos

\begin{tabular}{|c|c|c|c|c|c|c|c|}
\hline \multirow[t]{2}{*}{ Variáveis } & & $\begin{array}{c}\text { Sem } \\
\text { relacionamento }\end{array}$ & $\begin{array}{c}\text { Em } \\
\text { relacionamento }\end{array}$ & \multirow[t]{2}{*}{$\boldsymbol{U}$} & \multirow[t]{2}{*}{$Z$} & \multirow[t]{2}{*}{$p$} & \multirow[t]{2}{*}{$r$} \\
\hline & & $(n=323)$ & $(n=320)$ & & & & \\
\hline $\mathrm{DMS}-\mathrm{OM}$ & Posto médio & 328,85 & 315,19 & 49467,5 & $-0,94$ & 0,35 & 0,04 \\
\hline DMS - CM & Posto médio & 317,91 & 326,12 & 50360,5 & $-0,56$ & 0,57 & 0,02 \\
\hline SMAQ - I & Posto médio & 337,61 & 306,24 & 46637 & $-2,15$ & 0,03 & 0,08 \\
\hline SMAQ - VM & Posto médio & 339,02 & 304,82 & 46183,5 & $-2,37$ & 0,02 & 0,09 \\
\hline SMAQ -MM & Posto médio & 338,15 & 305,70 & 46462,5 & $-2,36$ & 0,02 & 0,09 \\
\hline MBIDS & Posto médio & 326,83 & 317,13 & 50121,5 & $-0,66$ & 0,51 & 0,03 \\
\hline SPAS & Posto médio & 324,46 & 319,51 & 50884,5 & $-0,34$ & 0,73 & 0,01 \\
\hline
\end{tabular}

Nota: DMS - OM: fator Orientação à Musculatura da Drive for Muscularity Scale; DMS - CM: fator Comportamentos relacionados ao Desenvolvimento da Musculatura da Drive for Muscularity Scale; SMAQ - I: fator Investimento na Musculatura do Swansea Muscularity Attitudes Questionnaire; SMAQ - VM: fator Valor da Musculatura do Swansea Muscularity Attitudes Questionnaire; SMAQ-MM: fator Masculinidade e Muscularidade do Swansea Muscularity Attitudes Questionnaire; MBIDS: Masculine Body Ideal Distress Scale; SPAS: Social Physique Anxiety Scale.

Tabela 3. Diferença entre grupos: seguros vs. inseguros financeiramente

\begin{tabular}{|c|c|c|c|c|c|c|c|}
\hline \multirow{2}{*}{ Variáveis } & & Inseguro & Seguro & \multirow{2}{*}{$\boldsymbol{U}$} & \multirow{2}{*}{$Z$} & \multirow{2}{*}{$p$} & \multirow{2}{*}{$r$} \\
\hline & & $(n=247)$ & $(n=395)$ & & & & \\
\hline DMS - OM & Posto médio & 364,62 & 294,54 & 38131,5 & $-4,66$ & $\mathrm{p}<0,001$ & 0,18 \\
\hline $\mathrm{DMS}-\mathrm{CM}$ & Posto médio & 321,37 & 321,58 & 48751,5 & $-0,14$ & 0,99 & 0,01 \\
\hline SMAQ - I & Posto médio & 337,62 & 311,24 & 44800,5 & $-1,75$ & 0,08 & 0,07 \\
\hline SMAQ - VM & Posto médio & 349,72 & 303,85 & 41811 & $-3,10$ & 0,002 & 0,12 \\
\hline SMAQ -MM & Posto médio & 339,76 & 310,08 & 44273 & $-2,10$ & 0,03 & 0,08 \\
\hline MBIDS & Posto médio & 323,52 & 320,23 & 48282,5 & $-0,22$ & 0,83 & 0,01 \\
\hline SPAS & Posto médio & 353,40 & 301,55 & 40902,5 & $-3,45$ & 0,001 & 0,13 \\
\hline
\end{tabular}

Nota: DMS - OM: fator Orientação à Musculatura da Drive for Muscularity Scale; DMS - CM: fator Comportamentos relacionados ao Desenvolvimento da Musculatura da Drive for Muscularity Scale; SMAQ - I: fator Investimento na Musculatura do Swansea Muscularity Attitudes Questionnaire; SMAQ - VM: fator Valor da Musculatura do Swansea Muscularity Attitudes Questionnaire; SMAQ-MM: fator Masculinidade e Muscularidade do Swansea Muscularity Attitudes Questionnaire; MBIDS: Masculine Body Ideal Distress Scale; SPAS: Social Physique Anxiety Scale.

\section{Diferenças entre grupos: ingressantes vs. veteranos}

As diferenças e similaridades encontradas, até então, a respeito do status do envolvimento amoroso e da segurança financeira dizem respeito aos homens, de uma forma geral. Todavia, as diferenças nesses grupos específicos, de veteranos e ingressantes, são mais específicas do ambiente militar. Devido às mudanças causadas pela rotina militar, pelo treinamento físico militar, pela necessidade de absorver em sua própria identidade aspectos da identidade militar, é esperado que algumas diferenças surjam entre os grupos.

Os resultados mostraram diferenças significantes no fator DMS-OM ( $U=48116 ; p=0,04, r=0,08)$, no fator SMAQ-I $(\mathrm{U}=46956 ; \mathrm{p}=0,01 ; \mathrm{r}=0,10)$ e no escore da SPAS $(\mathrm{U}=44468,5$; $\mathrm{p}<0,001 ; \mathrm{r}=0,14)$. Nas demais variáveis, não houve diferenças significantes entre aqueles que estavam ingressando no curso e entre os veteranos. Todavia, chamamos a atenção para o fato de que os resultados encontrados para o fator DMSOM e o escore da MBIDS estão muito próximos da zona de 
Tabela 4. Diferença entre grupos: veteranos vs. ingressantes

\begin{tabular}{|c|c|c|c|c|c|c|c|}
\hline \multirow{2}{*}{ Variáveis } & & Veteranos & Ingressantes & \multirow{2}{*}{$\boldsymbol{U}$} & \multirow{2}{*}{$Z$} & \multirow{2}{*}{$p$} & \multirow{2}{*}{$r$} \\
\hline & & $(n=326)$ & $(n=326)$ & & & & \\
\hline DMS - OM & Posto médio & 313,43 & 339,57 & 48877,5 & $-1,77$ & 0,08 & 0,07 \\
\hline $\mathrm{DMS}-\mathrm{CM}$ & Posto médio & 341,90 & 311,10 & 48116 & $-2,10$ & 0,03 & 0,08 \\
\hline SMAQ - I & Posto médio & 307,54 & 345,46 & 46956 & $-2,58$ & 0,01 & 0,10 \\
\hline SMAQ - VM & Posto médio & 320,32 & 332,68 & 51122 & $-0,85$ & 0,39 & 0,03 \\
\hline SMAQ -MM & Posto médio & 329,23 & 323,77 & 52247 & $-0,40$ & 0,69 & 0,01 \\
\hline MBIDS & Posto médio & 340,02 & 312,98 & 48729 & $-1,84$ & 0,06 & 0,07 \\
\hline SPAS & Posto médio & 299,91 & 353,09 & 44468,5 & $-3,61$ & $<0,001$ & 0,14 \\
\hline
\end{tabular}

Nota: DMS - OM: fator Orientação à Musculatura da Drive for Muscularity Scale; DMS - CM: fator Comportamentos relacionados ao Desenvolvimento da Musculatura da Drive for Muscularity Scale; SMAQ - I: fator Investimento na Musculatura do Swansea Muscularity Attitudes Questionnaire; SMAQ - VM: fator Valor da Musculatura do Swansea Muscularity Attitudes Questionnaire; SMAQ-MM: fator Masculinidade e Muscularidade do Swansea Muscularity Attitudes Questionnaire; MBIDS: Masculine Body Ideal Distress Scale; SPAS: Social Physique Anxiety Scale.

rejeição da hipótese nula (ver Tabela 4). Reporta-se, ainda, que há um maior número de pessoas seguras financeiramente $[\chi 2(1,642)=15,50 ; p<0,001]$, um maior número de pessoas em relacionamento amoroso $[\chi 2(1,643)=22,78 ; p<0,001]$ e um maior número de pessoas fisicamente ativas $[\chi 2(1$, $642)=28,75 ; \mathrm{p}<0,001]$ entre os alunos veteranos.

\section{Discussão}

O objetivo deste trabalho exploratório foi fornecer mais dados a respeito de drive for muscularity no Brasil, especificamente no Exército Brasileiro. As evidências aqui geradas sugerem a existência de associações estatisticamente significantes entre as variáveis de drive for muscularity e os hábitos de prática de atividade física, satisfação com a vida e com o corpo e ansiedade físico-social. Ademais, quanto ao status de relacionamento amoroso, foram encontradas diferenças nos escores do fator SMAQ-I, SMAQ-VM e SMAQ-MM. O escore da SPAS e do fator DMS-OM foram estatisticamente diferentes, nesta amostra, em relação à percepção da segurança financeira e ao tempo no ambiente militar. Ainda para este último, foi encontrada diferença em relação ao escore da SMAQ-I. Já para a variável percepção da segurança financeira, além das já anteriormente mencionadas, foram verificadas diferenças em relação aos escores da SMAQ-VM e SMAQ-MM.

Estudos anteriores já haviam demonstrado haver uma associação positiva entre o engajamento em exercício resistido e drive for muscularity (McCreary \& Sasse, 2000, Ricciardelli \& McCabe, 2004). No presente estudo, essas evidências suportam a interdependência encontrada, por meio da análise de clusters, entre as variáveis caracterizadoras do exercício físico (intensidade, duração e frequência) e os fatores SMAQ-VM e SMAQ-MM. Esses resultados evidenciam que os efeitos da atividade física sobre drive for muscularity são mais pontuais, já que apenas esses aspectos específicos compartilham similaridade com as características do exercício físico praticada pelos respondentes.

A satisfação com a vida, a satisfação com o corpo e o fator SMAQ-I formaram um segundo cluster. Teoricamente, essa interdependência tem sentido no contexto do grupo que foi analisado, pois o sucesso profissional tem impacto na satisfação com o corpo (Smith, Li \& Joiner, 2011) e na satisfação com a vida (Leung, Cheung, \& Liu, 2011), inclusive no contexto militar (Proyer, Annen, Eggimann, Scheneider, \& Ruch, 2012). Na carreira militar, espera-se um comprometimento na manutenção e melhora da forma física para a execução das missões, que de forma subliminar, têm implicação nas possibilidades de promoção na carreira militar (Ministério da Defesa, 2010). Os resultados aqui encontrados são evidências de que o investimento na musculatura é o componente de drive for muscularity que tem mais influência na satisfação com a vida e com o corpo.

Identificou-se, ainda, um terceiro cluster, formado pelas similaridades encontradas entre o escore da SPAS, da MBIDS e os fatores DMS-OM e DMS-CM. Consegue-se interpretar esse cluster quando se considera que os militares, ao longo de sua carreira, são submetidos à avaliação de desempenho físico, a fim de verificar seu condicionamento físico. A ansiedade físico-social é um tipo de ansiedade que pode-se vivenciar em resposta à avaliação de uma outra pessoa sobre a sua aparência/seu físico (Hart et al., 1989), ou seja, refere-se de forma específica à ansiedade sentida quando a aparência e as funções corporais são analisadas. As avaliações da aptidão física, regularmente conduzidas ao longo da carreira militar, são o cenário de fundo propício para a vivência da ansiedade físico social. Além dessas interpretações teóricas, o cluster pode ser também suportado por associações moderadas previamente encontradas entre os fatores da DMS e a SPAS (McCreary \& Saucier, 2009; Nowell \& Ricciardelli, 2008) e entre o escore da MBIDS (Campana et al., 2013).

As associações entre as variáveis apresentaram-se de forma distinta entre os ingressantes e os veteranos em alguns aspectos. Há uma diferença na intensidade da associação 
entre a ansiedade físico social e os fatores de drive for muscularity investigados, sendo esta fraca nos ingressantes e moderada nos veteranos. É possível que as avaliações de desempenho físico periódicas e a necessidade de ter um corpo que atenda os padrões das missões sejam o fator subjacente que explique essa diferença de força entre as correlações. Porém, isso não significa que a prática de exercício físico não possa ter um efeito positivo na diminuição dos níveis de ansiedade físico-social, como já observado anteriormente (Ginis, Eng, Arbour, Hartman, \& Phillips, 2005). De fato, nossos resultados apontaram que os militares veteranos têm menores índices de ansiedade físico-social quando comparado aos ingressantes. O que esses dois resultados em conjunto apontam é que nos militares veteranos, pode sim haver uma diminuição da ansiedade físico-social, mas que esta passa a estar mais intrinsicamente associada ao valor, ao investimento e à orientação à musculatura. Uma pesquisa futura poderá confirmar essa argumentação.

É importante também comentar a ausência de associação entre as variáveis do drive for muscularity e ansiedade físicosocial com as variáveis caracterizadoras do exercício físico na amostra de militares veteranos, em oposição às correlações de moderadas a fracas encontradas entre as mesmas variáveis no grupo de militares ingressantes. Podemos interpretar essa diferença à luz de uma provável mudança de atitude frente ao exercício físico. Para os veteranos, o exercício físico aproxima-se muito mais de uma rotina de trabalho necessária, enquanto para os ingressantes pode ser ainda uma escolha no estilo de vida. Essa interpretação explica ainda as diferenças na associação das variáveis do drive for muscularity e ansiedade físico-social e a satisfação com a vida, se forem associadas às argumentações anteriores sobre a importância do corpo musculoso na carreira militar, o sucesso profissional e sua implicação na satisfação com a vida. Essa interpretação é especulativa, porém crível, mas deverá ser comprovada numa pesquisa futura.

A capacidade de manter o papel de provedor e a confiança de que poderá fazê-lo por um longo tempo têm sido associadas a traços da imagem corporal como satisfação com o corpo (Smith et al., 2011). Nossos resultados sugerem que a percepção de segurança financeira provoca diferenças significantes em traços específicos do drive for muscularity, como DMS-OM, SMAQ-VM, SMAQ-MM, além da ansiedade físico-social. Evidências anteriores já sugeriam haver diferença em aspectos psicossociais entre pessoas seguras e inseguras financeiramente (Graetz, 1993; Jones, 1991; Swami, Kannan \& Furnhan, 2012; Warr, 1987) e nossos resultados são mais uma confirmação da influência da seguridade financeira no bem-estar e na identidade do sujeito.

Contrário ao que foi verificado por Campana et al. (2013), foram observadas variações estatisticamente significantes nos dois status de relacionamento amoroso: comprometidos (que incluiu pessoas casadas, aqueles que estão noivos e namoradando) e não comprometidos. Esses resultados adicionam novas evidências, específicas de drive for muscularity, às pesquisas que apontam que homens que estão em um relacionamento amoroso, sejam eles namorados, noivos ou casados, têm um padrão maior de satisfação com o corpo e menor direcionamento à aparência (Giles \& Close, 2008; Luciano, 2001).
Quanto às diferenças entre militares veteranos e ingressantes no Curso de Sargento do Exército, destacam-se os maiores níveis de comportamento para ficar forte entre os veteranos, explicado pela própria rotina de treinamento do Exército, já aludida neste estudo, e os maiores níveis de investimento na musculatura entre os ingressantes. Quanto a este último, argumenta-se que a entrada do aluno no curso pode aumentar a atenção ao corpo musculoso, para atender à aparência viril e à função nas missões, em ordem de ser um bom militar (Morgado, 2012). Mas, o que mais se destaca na comparação desses dois grupos, é a ausência de diferenças na maioria das variáveis observadas, indicando que o impacto da rotina militar é bem específico nos traços do drive for muscularity. Adicionalmente, tem-se que considerar que a importância da aparência do corpo musculoso é um valor muito difundido entre os homens brasileiros, presente em diferentes classes sociais e funções laborais (Goldenberg, 2002; Sabino, 2000), e por isso, espera-se certa homogeneidade em relação ao drive for muscularity.

No presente estudo, há limitações que precisam ser mencionadas. Nossos resultados não devem ser generalizados, dada a natureza não-probabilística e não representativa de nossa amostra. Em decorrência da natureza não paramétrica de nossos dados, foi possível estabelecer de forma interpretativa relações de proximidade entre os constructos, através de análise de cluster, mas uma análise de regressão ou uma modelagem de equações estruturais é necessária para estabelecer de forma mais confirmativa as relações de dependência entre os construtos aqui estudados. Adicionalmente, há a necessidade de investigar a extensão das relações e associações entre os traços de drive for muscularity e outros construtos relevantes, como, por exemplo, satisfação com o corpo, uso de esteróides anabólicos, dependência do exercício, atratividade física, felicidade conjugal e satisfação com o trabalho, no contexto brasileiro. De igual forma, associar e diferenciar variáveis antropométricas e indicadores de desempenho físico, como níveis de força máxima, condicionamento aeróbico, resistência de força de membros inferiores e membros superiores, são análises importantes não contempladas neste trabalho.

Apesar das limitações apontadas, este trabalho, de natureza eminentemente exploratória, trouxe novas evidências, interpretações e especulações para serem investigadas em pesquisas futuras, colaborando para um entendimento mais ampliado de drive for muscularity em nosso país, mais especificamente no contexto militar, onde o corpo forte e viril é importante para a segurança e sucesso do profissional militar. Por esta importância, seria relevante acompanhar os níveis de drive for muscularity no Exército.

\section{Referências}

Bahrke, M. S. (2007). Muscle enhancing substances and strategies. In J. K. Thompson \& G. Cafri (Eds.), The muscular ideal: Psychological, social and medical perspectives (pp.141-160). Washington, DC: APA. 
Campana, A. N. B. C., Tavares, M. C. G. C. F., \& Silva, D. (2011, julho). Tradução, adaptação cultural e validação da Social Physique Anxiety Scale, para a Língua Portuguesa no Brasil. Actas do 70 Congresso Iberoamericano da Avaliação Psicológica, Lisboa, Portugal.

Campana, A. N. B. C., Tavares, M. C. G. C. F., Swami, V., \& Silva, D. (2013). An examination of the psychometric properties of Brazilian Portuguese translations of the drive for muscularity scale, the Swansea Muscularity Attitudes Questionnaire, and the Masculine Body Ideal Distress Scale. Psychology of Men \& Masculinity.14, 376-388

Carlton, J. R., Manos, G. H, \& Van Slyke, J. A. (2005). Anxiety and abnormal eating behavior associated with cyclical readiness testing in a naval hospital active duty population. Military Medicine, 170, 663-667

Edwards, S., \& Launder, C. (2000). Investigating muscularity concerns in male body image: Development of the Swansea Muscularity Attitudes Questionnaire. International Journal of Eating Disorders, 28, 120-124.

Estado-Maior do Exército (EME). Manual de Campanha C 2020 - Treinamento Físico Militar ( $3^{\mathrm{a}}$ Ed). Brasília: Exército Brasileiro, 2002.

Estado-Maior do Exército (EME). Diretriz para o Treinamento Físico Militar do Exército e sua Avaliação. Brasília: Exército Brasileiro, 2008.

Giles, D. C., \& Close, J. (2008). Exposure to 'lad magazines' and drive for muscularity in dating and non-dating young men. Personality and Individual Differences, 44, 1610-1616.

Ginis, K. A. M., Eng, J. J., Arbour, K. P., Hartman, J. W., \& Phillips, S. M. (2005). Mind over muscle? Sex differences in the relationship between body image change and subjective and objective physical changes following a 12-week strengthtraining program. Body Image, 2, 363-372.

Goldenberg, M. (2002). Nu \& vestido. Rio de Janeiro: Record.

Graetz, B. (1993). Health consequences of employment and unemployment: Longitudinal evidence for young men and women. Social Science \& Medicine, 36, 715-724.

Gunderson, E. K. E. (1965). Body size, self-evaluation, and military effectiveness. Journal of Personality and Social Psychology, 2, 902-906.

Hair, J. F., Jr., Anderson, R. E., Tatham, R. L., \& Black, W. B. (2009). Multivariate data analysis. Upper Saddle River, NJ: Prentice Hall.

Hart, E., Leary, M. R., \& Rejesky, W. J. (1989). The measurement of social physique anxiety. Journal of Sport and Exercise Psychology, 11, 94-104.

Jones, L. (1991). The health consequences of economic recessions. Journal of Health \& Social Policy, 3, 1-14.

Kimmel, S. B., \& Mahalik, J. R. (2004). Measuring masculine body ideal distress: Development of a measure. International Journal of Men's Health, 3, 1-10.

Leung, A. S. M., Cheung, Y. A., \& Liu, X. (2011). The relations between life domain satisfaction and subjective well-being. Journal of Managerial Psychology, 26, 155-169.

Luciano, L. (2001). Looking good: Male body image in modern America. New York: Hill and Wang.

Malhotra, N. K. (1999). Marketing research: An applied orientation. New Jersey: Prentice Hall.
Magnani, R. (1999). Sampling guide. IMPACT Food Security and Nutrition Monitoring Project. Arlington: International Science and Technology Institute.

McCreary, D. R. (2011). Body image and muscularity. In T. Cash \& L. Smolak (Eds.), Body image: A handbook of science, practice, and prevention (pp. 198-205). New York: Guilford.

McCreary, D. R., \& Sasse, D. K. (2000). An exploration of the drive for muscularity in adolescent boys and girls. Journal of American College Health, 48, 297-304.

McCreary, D. R., Sasse, D. K., Saucier, D. M., \& Dorsch, K. D. (2004). Measuring the drive for muscularity: Factorial validity of the Drive for Muscularity Scale in men and women. Psychology of Men and Masculinity, 5, 49-58.

McCreary, D. R., \& Saucier, D. M. (2009). Drive for muscularity, body comparison, and social physique anxiety in men and women. Body Image, 6, 24-30.

McNulty, P. A. (1997). Prevalence and contributing factors of eating disorder behaviors in active duty Navy men. Military Medicine, 162, 753-8.

Ministério da Defesa (2010). Módulo didático para execução e avaliação do treinamento físico militar para o curso de formação de oficiais da escola de formação de oficiais do Exército. Rio de Janeiro: Exército Brasileiro.

Morgado, J. J. M. (2012). Imagem corporal de homens militares. (Unpublished master's thesis), Universidade Federal de Juiz de Fora, Juiz de Fora, MG.

Morrison, T. G., Morrison, M. A., \& Mc Cann, L. (2006). Striving for bodily perfection? An overview of the drive for muscularity. In M. Kindes (Ed.), Body image: New research (pp. 1-34). Columbus, NY: Nova Science.

Neves, E. B., \& Mello, M. G. S. (2009). O risco da profissão militar na cidade do Rio de Janeiro em "tempo de paz": a percepção da tropa. Ciência e Saúde Coletiva, 14, 1669-1707.

Nowell, C., \& Ricciardelli, L. A. (2008). Appearance-based comments, body dissatisfaction, and drive for muscularity in males. Body Image, 5, 337-345.

Olivardia, R. (2007). Muscle dysmorphia: Characteristics, assessment, and treatment. In K. Thompson \& G. Cafri (Eds.), The muscular ideal: Psychological, social and medical perspectives (pp.123-140). Washington: APA.

Pope, H. G., Phillips, K. A., \& Olivardia, R. (2000). The adonis complex. New York: Free Press.

Pope, H. G., Gruber, A. J., Choi, P., Olivardia, R., \& Phillips, K. A. (1997). Muscle dysmorphia: an unrecognized form of body dismorphic disorder. Psychosomatics, 38, 548-557.

Pope, C. G., Pope, H. G., Menard, W., Fay, C., Olivardia, R., \& Phillips, K. A. (2005). Clinical features of muscle dysmorphia among males with body dysmorphic disorder. Body Image, 2, 395-400.

Proyer, R. T., Annen, H., Eggimann, N., Schneider, A., \& Ruch, W. (2012). Assessing the "good life" in a military context: How does life and work satisfaction relate to orientations to happiness and career success among Swiss professional officers? Social Indicators Research, 106, 577-590.

Ricciardelli, L. A., \& McCabe, M. P. (2004). A biopsychosocial model of disordered eating and the pursuit of muscularity in adolescent boys. Psychological Bulletin, 130, 179-205. 
Sabino, C. (2000). Musculação: expansão e manutenção da masculinidade. In M. Goldenberg (Ed.), Os novos desejos: das academias de musculação às agências de encontros (pp. 61-104). Rio de Janeiro: Record.

Smith, A. R., Li, N., \& Joiner, T. E. (2011). The pursuit of success: Can status aspirations negatively affect body satisfaction? Journal of Social and Clinical Psychology, 30, 531-547.
Swami, V., Kannan, K., \& Furnham, A. (2012). Positive body image: Inter-ethnic and rural-urban differences among an indigenous sample from Malaysian Borneo. International Journal of Social Psychiatry, 58, 568-576.

Warr, P. (1987). Work, unemployment, and mental health. New York: Oxford University Press.

\section{CONGRESSO BRASILEIRO DE PSICOTERAPIAS CORPORAIS}

$22 / 08$ a $24 / 08 / 2014$

Curitiba - PR

Hotel Pestana

www.centroreichiano.com.br/ 